\title{
Anti-CD33 Antigen/CD3 Receptor Bispecific Monoclonal Antibody AMV564
}

National Cancer Institute

\section{Source}

National Cancer Institute. Anti-CD33 Antigen/CD3 Receptor Bispecific Monoclonal

Antibody AMV564. NCI Thesaurus. Code C135632.

An anti-CD33/anti-CD3 bispecific tetravalent antibody, with potential immunostimulatory and antineoplastic activities. Anti-CD33/CD3 tetravalent bispecific monoclonal antibody AMV564 possesses two antigen-recognition and binding sites, one for the CD3 complex, a group of T-cell surface glycoproteins that complex with the T-cell receptor (TCR), and one for CD33, a tumor-associated antigen (TAA) overexpressed on the surface of a variety of tumor cell types. Upon infusion of AMV564, this bispecific antibody binds to CD3-expressing T-cells and CD33-expressing tumor cells, thereby crosslinking CD33expressing tumor cells and cytotoxic T-lymphocytes (CTLS). This may result in a potent CT L-mediated cell lysis of CD33-expressing cells. CD33, a glycoprotein expressed by a variety of cancers, including the majority of acute myeloid leukemias (AMLs), and normal non-pluripotent hematopoietic stem cells, plays a key role in tumor initiation, proliferation and progression. 